\title{
Neoadjuvant chemoradiotherapy followed by liver transplantation is a promising treatment for patients with unresectable hilar cholangiocarcinoma: A case report
}

\author{
JUN WANG $^{1 *}$, HALMURAT OBULKASIM ${ }^{1 *}$, XIAOPING ZOU ${ }^{2}$, BAORUI LIU $^{3}$, \\ YAFU WU ${ }^{1}$, XINGYU WU ${ }^{1}$ and YITAO DING ${ }^{1}$ \\ Departments of ${ }^{1}$ Hepatobiliary Surgery, ${ }^{2}$ Gastroenterology and \\ ${ }^{3}$ Oncology, Affiliated Drum Tower Hospital of Nanjing University Medical School, \\ Nanjing, Jiangsu 210008, P.R. China
}

Received August 3, 2015; Accepted August 19, 2016

DOI: $10.3892 / \mathrm{ol} .2018 .9878$

\begin{abstract}
Hilar cholangiocarcinoma is a highly malignant tumor and is currently treated by surgical resection or liver transplantation; however, these treatments result in poor patient prognosis accompanied with high recurrence and low patient mortality rates. Neoadjuvant therapy with liver transplantation is a novel treatment that exhibits promising clinical application, with a reported 5-year survival rate of $82 \%$. However, transplantation centers conducting research into this treatment are limited due to its length and complexity. In the current study, the effects of brachytherapy and chemoradiotherapy followed by orthotopic liver transplantation (OLT) were investigated in a patient with unresectable hilar cholangiocarcinoma. Following treatment, the liver function of the patient normalized and physical status significantly improved. Furthermore, tomographic evaluation demonstrated no sign of recurrence 8 months later following continued adjunct chemotherapy. Therefore, neoadjuvant therapy followed by OLT may be an effective novel therapeutic strategy to treat patients with unresectable hilar cholangiocarcinoma.
\end{abstract}

\section{Introduction}

Cholangiocarcinoma arises from malignantly transformed bile duct epithelium. Despite only accounting for $10 \%$ of all forms of primary hepatobiliary cancer, its prevalence has

Correspondence to: Professor Yitao Ding, Department of Hepatobiliary Surgery, Affiliated Drum Tower Hospital of Nanjing University Medical School, 321 Zhongshan Road, Nanjing, Jiangsu 210008, P.R. China

E-mail: dingyitao@yeah.net

*Contributed equally

Key words: neoadjuvant chemoradiotherapy, liver transplantation, unresectable hilar cholangiocarcinoma, brachytherapy, steroid early withdrawal increased in recent decades (1). Hilar cholangiocarcinoma, also known as Klatskin tumors, represents $60-70 \%$ of all cholangiocarcinoma cases and constitutes a major surgical challenge as it is characteristically located in the vicinity of pivotal structures (2).

Currently, surgical resection offers the best treatment outcome, however prognosis remains dismal with a 5-year survival rate of $9-28 \%$ (3). The subset of patients with hilar cholangiocarcinoma, however, are not suitable for resection due to advanced tumor stage or the presence of underlying liver disease, including primary sclerosis cholangitis (PSC) (4). Orthotopic liver transplantation (OLT) has been proposed as an alternative to treat patients with unresectable hilar cholangiocarcinoma (5), as it may increase the resection margin and circumvent subsequent liver failure that occurs due to insufficient hepatic portions or co-morbidity (6). However, interest in OLT has waned due to its high recurrence rate, and patient prognosis following OLT remains poor $(7,8)$; therefore, more efficient therapeutic strategies are required (9).

Neoadjuvant chemoradiation plus OLT, which has been accomplished by the Mayo Clinic, demonstrates promising results regarding patient prognosis, with a 5-year survive rate of $\sim 82 \%$ (10). Encouraged by these outcomes, the United Network of Organ Sharing/Organ Procurement and Transplantation Network approved the allocation of a standard model of end-stage liver disease exception score for patients with hilar cholangiocarcinoma who undergo this treatment modality (11).

The neoadjuvant chemoradiotherapy method, also known as the 'Mayo protocol', is currently regarded as having therapeutic applications for hilar cholangiocarcinoma. However, its efficacy and safety have been questioned as only a few treatment centers have investigated its effects and all studies have involved $<300$ patients (12). This is attributed, at least in part, to protocol complexity and completion difficulty, and the involvement of multiple departments.

The current case aims to build on the findings of the Mayo Clinic and describes a patient with unresectable hilar cholangiocarcinoma, who underwent brachytherapy and chemoradiotherapy followed by OLT, and remains disease-free after 8 months. 


\section{Case report}

A 53-year-old woman presented with yellow skin and urine upon admittance to the Department of Hepatobiliary Surgery, Nanjing Drum Tower Hospital, Affiliated Hospital of Nanjing University Medical School (Nanjing, China) on March 18, 2013. Computed tomography (CT) and magnetic resonance imaging (MRI) revealed a hilar mass with left and right hepatic duct and vessel involvement (Figs. 1A and B). Subsequent magnetic resonance cholangiopancreatography exhibited disruption of the hepatic bile duct (Fig. 1C). The total bilirubin carbohydrate antigen (CA)19-9 levels were $479.6 \mu \mathrm{mol} / 1$ and $1,044 \mathrm{U} / \mathrm{ml}$, respectively, which were beyond the healthy ranges (3-25 $\mu \mathrm{mol} / 1$ and $0-37 \mathrm{U} / \mathrm{ml}$, respectively).

To alleviate jaundice of the patient, two tubes were placed in the left and right bile duct using the percutaneous transhepatic drainage (PTCD) technique. The daily drainage volume was $300 \mathrm{ml}$. The patient was classed as having type IV cholangiocarcinoma (Bismuth classification) (13) with invasion of major vessels; therefore, a radical, major hepatectomy was considered to be unfeasible. Inspired by the success of the Mayo clinic, it was decided that neoadjuvant therapy plus OLT was the best treatment option.

Chest and abdominal MRI, contrast-enhanced CT, a bone scan and positron emission tomography (PET)-CT were employed to rule out local lymph node and distant metastasis. Repetitive endoscopic retrograde cholangiopancreatography (ERCP) was performed to obtain positive results by brush cytology, which indicated the presence of malignant cells consistent with the patient diagnosis (Fig. 1D). Notably, the patient had developed a number of severe, but non-lethal complications, including acute cholangitis, liver abscess and biliary-pleural fistula, due to repeated ERCP and cholangiography (Fig. 2A). Administering anti-infection, drainage and nutrition support alleviated these symptoms (Fig. 2B).

I-125 radioactive plastic stents were used to perform brachytherapy (Fig. 3A). Each stent was composed of a drainage tube with a stab at each end, which had a stabilizing effect. Two opposite channels were made in the wall of stents paralleling the drainage lumen, with an irradiation window in the lateral wall of the channel. The channel diameter was slightly smaller than that of the radioactive seeds, as were the length and width of the irradiation windows. This immobilizes the radioactive seeds and improve the effectiveness of radiation treatment. The radioactive I-125 seeds (Shanghai Xinke Pharmaceutical Co., Ltd., Shanghai, China) were $4.5 \mathrm{~mm}$ long and $0.8 \mathrm{~mm}$ thick, with a half-life of 60.1 days.

Nasobiliary radiography was performed to determine the extent of bile duct involvement (Fig. 3B). A total of 11 radioactive seeds were loaded into the channel in sequence and the ERCP procedure was performed to put stents in the appropriate location on May 7, 2013 (Fig. 3C). Following 1 month, external beam radiotherapy was administrated at a targeted dose of 30 Gy (30 fractions of 1.5 Gy twice a day). Concomitantly, intravenous capecitabine was provided at $1.0 \mathrm{~g}$ for 2 weeks, and repeated until 14 days post-transplantation. On July 26, 2013, the patient received a liver allograft from a deceased donor. However, the patient underwent laparoscopic exploration at the beginning of liver transplantation to assess for lymph node and distant metastases, which were negative. A standard
OLT was performed with no major incidents (Figs. 4A and B). Immunosuppressive therapy was provided with tacrolimus, mycophenolate and corticosteroids. Corticosteroids were withdrawn a week after OLT was completed.

Subsequent specimen pathology revealed an adenosquamous cell carcinoma, measuring $4 \times 2.5 \times 2.5 \mathrm{~cm}$, with focal necrosis. The tumor was classified as tumor-node-metastasis (TNM) stage II $\left(\mathrm{T}_{2 \mathrm{~b}} \mathrm{~N}_{0} \mathrm{cM}_{0}\right)$ due to neural and vascular invasion (Fig. 4C). Following treatment, liver function normalized and physical status improved significantly (Table I). Follow-up tomographic evaluation 8 months post-transplantation indicated that the liver was normal without tumor recurrence (Fig. 5). Three chemotherapy cycles were administered $\left(1.0 \mathrm{mg} / \mathrm{m}^{2}\right.$ capecitabine twice daily) 1 month after OLT. Each chemotherapy cycle was continued for 3 weeks.

\section{Discussion}

Although hilar cholangiocarcinoma is a debilitating disease with poor patient prognosis, there is interest in improving the efficacy and safety of the currently limited treatment options available. A number of patients are diagnosed during an advanced cancer stage, which deprives them of the opportunity to undergo resection (14). Furthermore, the survival rate of those eligible to undergo surgical resection remains low, suggesting that resection is not an acceptable treatment for hilar cholangiocarcinoma (15-17). It was previously considered that OLT alone may be an effective method of treating cholangiocarcinoma, however, it was later contraindicated due to the high recurrence and low survival rates accompanying its use. Therefore, reducing the recurrence rate has become a key objective.

Surgeons suggested the use of OLT plus Whipple's operation to eradicate tumors, as the loco-region recurrence rate is high (18). Unfortunately, this approach did not significantly improve the poor survival rate, and tumor recurrence continued to account for the majority of patient mortalities (19). In an attempt to achieve a more radical margin, the Starzl group proposed the concept of cluster-OLT, which involves the removal of all visceral organs derived from the foregut and part of the colon (20). This aggressive method resulted in markedly high post-operative mortality and no significant improvements in the long term survival rate of patients (20).

Neoadjuvant therapy refers to adjuvant chemoradiation performed prior to surgery. This therapy aims to shrink inoperable tumors to make surgery more feasible, eradicate micro metastatic sites and reduce tumor cell viability to minimize the possibility of metastasis. Neoadjuvant therapy has been widely used in different types of cancer, including breast (21) and gastrointestinal tumors (22), with great success, and was first introduced by Nebraska University to treat cholangiocarcinoma in combination with liver transplantation (23). The neoadjuvant protocol consists of biliary brachytherapy and intravenous infusion of 5-fluorouracil until transplantation. In the long-term, $45 \%$ of patients that underwent this procedure were reported to be tumor-free post-transplantation, demonstrating significant progress in cholangiocarcinoma prognosis (23).

The Mayo Clinic pioneered a neoadjuvant study by extensively investigating its clinical applications. In 2005, the study presented promising data with 1-3- and 5-year patient survival rates of 92,82 and $82 \%$, respectively, following neoadjuvant 
A

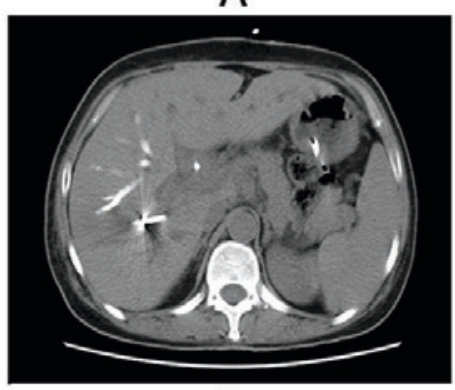

C

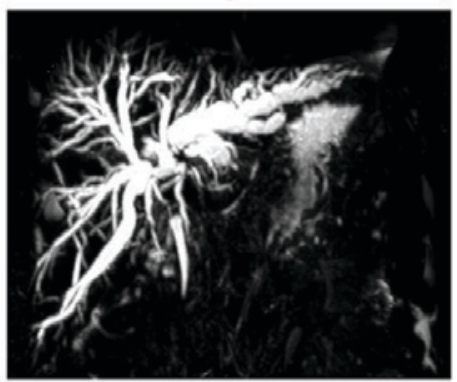

B

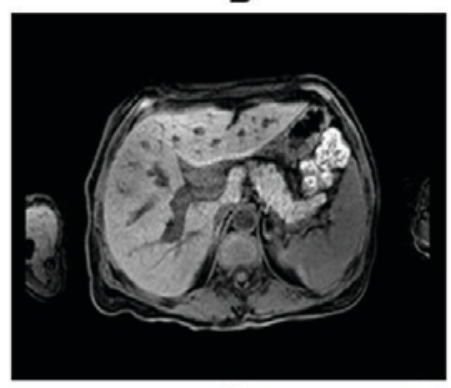

D

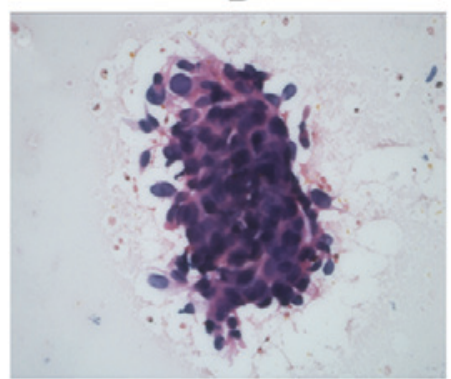

Figure 1. Various methods used to diagnose hilar cholangiocarcnoma. (A) Computed tomography, (B) magnetic resonance imaging, (C) magnetic resonance cholangiopancreatography and (D) brush cytology.

A

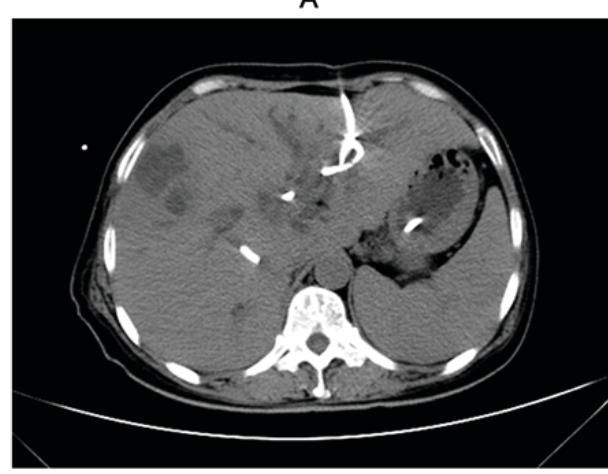

B

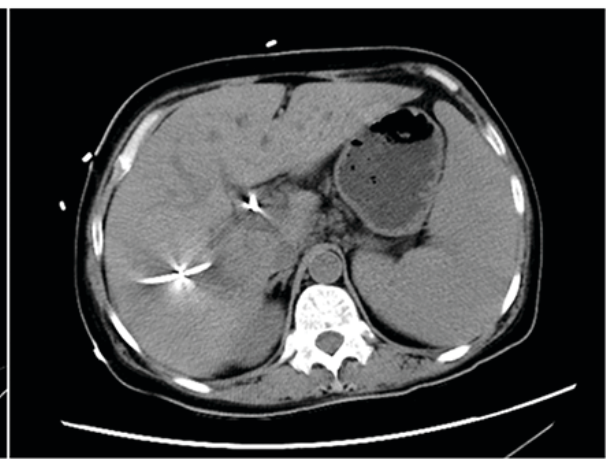

Figure 2. Hilar mass prior to and following treatment. (A) Hepatic abscess experienced by the patient prior to treatment and (B) the lesion resolved following treatment.

therapy plus OLT, which were markedly higher than the survival rates of patients with liver cancer following transplantation (10). The neoadjuvant protocol differed from that of Nebraska University by including external beam irradiation (10). The Mayo Clinic has published a series of articles concerning neoadjuvant plus liver transplantation for cholangiocarcinoma. In this ongoing study, patients with hilar cholangiocarcinoma who underwent neoadjuvant therapy were recommended for liver transplantation (24). Furthermore, Wu et al (18) conducted clinical research to determine if neoadjuvant plus OLT-Whipple therapy was able to improve patient prognosis. A total of 6 patients were enrolled and administered radiation only, including external beam irradiation and brachytherapy, prior to transplantation. Excellent results were achieved overall, with 5 patients recurrence-free at 10.1 years post-treatment, although 1 patient succumbed at 55 months post-transplant due to an unrelated cause (18). These results implied that a more aggressive approach of liver transplantation may improve long-term survival rates, provided that post-operative lethal complications are avoided.
The aforementioned studies focused on early stage hilar cholangiocarcinoma and patients with a clinical stage beyond II were excluded from liver transplantation. However, other previous studies have investigated possible therapeutic strategies to improve the prognosis of patients with advanced hilar cholangiocarcinoma. Researchers from the University of California, Los Angeles, investigated whether neoadjuvant therapy plus transplantation may be more beneficial to patients with advanced hilar cholangiocarcinoma compared with surgical resection alone (25). Locally advanced hilar cholangiocarcinoma was defined as follows: A tumor size $>3 \mathrm{~cm}$; invasion beyond the wall of the bile duct to the gallbladder, liver and either ipsilateral branches of the hepatic artery or the portal vein; or metastasis to the regional lymph nodes. Notably, neoadjuvant and adjuvant therapies in the liver transplantation group resulted in a 47\% 5-year recurrence-free survival rate, whereas patients who underwent resection alone had all succumbed to the disease 5 years on (25). Therefore, evidence from numerous studies suggests that neoadjuvant therapy plus OLT may be 


\section{A}

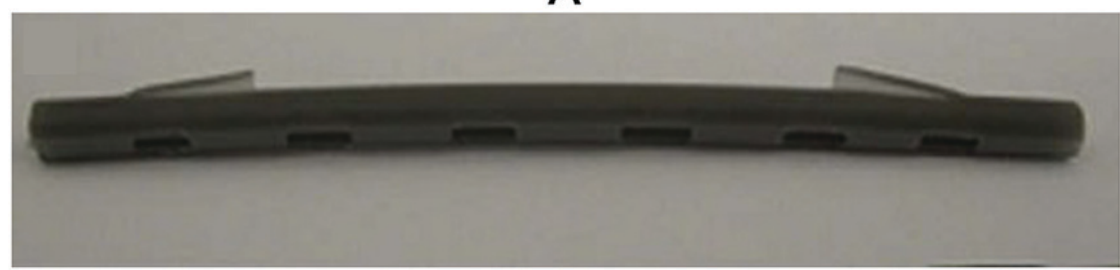

B

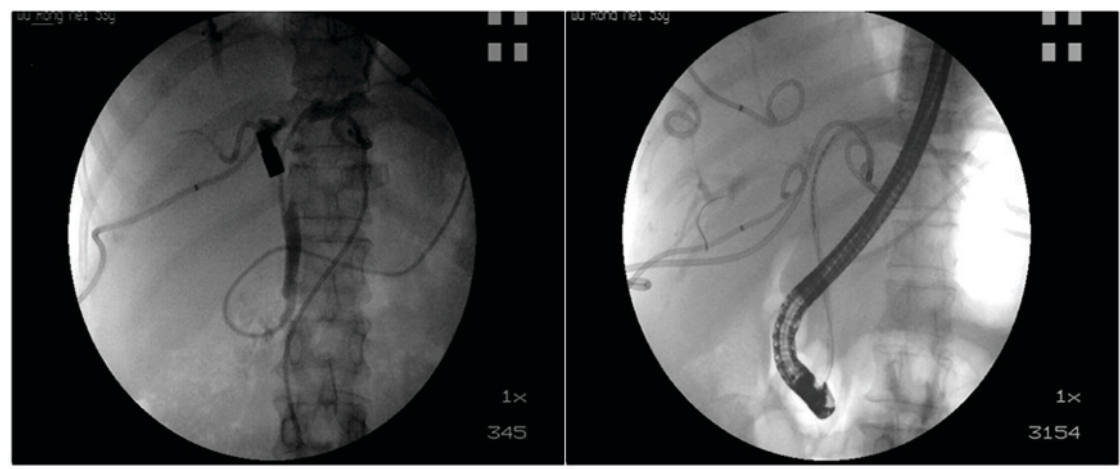

Figure 3. Performance of brachytherapy using a stent. (A) The stent used to conduct brachytherapy. (B) Nasobiliary image revealing the length of the bile duct lesion, which is $<5 \mathrm{~cm}$. (C) Location of the stent, in which 11 radioactive seeds were loaded.

A

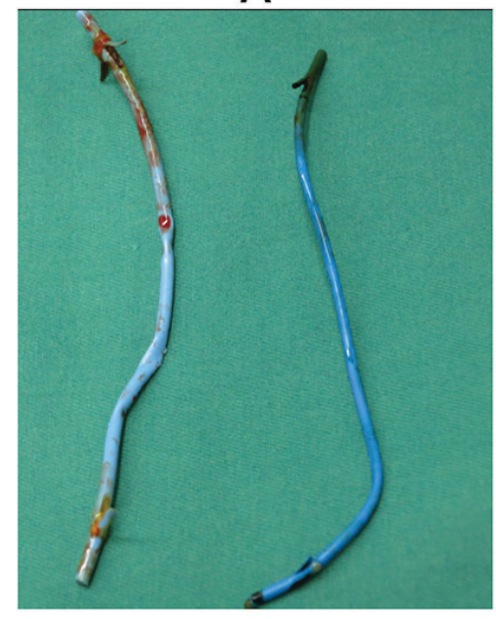

B

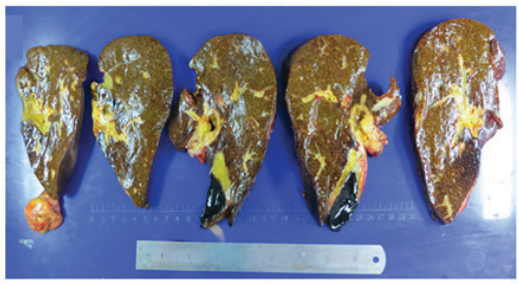

C

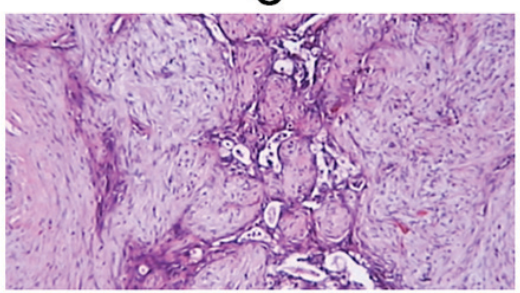

Figure 4. Effects of treatment on the hilar mass. (A) Image showing the retrieved radioactive stent during liver transplantation and (B) patient liver with hilar dissection following transplantation. (C) Histopathological image of a formalin-fixed biopsy sample from the liver, indicating adenosquamous cell carcinoma accompanied by vascular and neural invasion. Magnification, x200.

the optimal treatment for hilar cholangiocarcinoma, even for patients with an advanced stage of the disease (26-28).

Diagnosing hilar cholangiocarcinoma remains a challenge. With regards to the Mayo Clinic protocol, the clinical diagnosis of hilar cholangiocarcinoma requires the presence of a malignant-appearing stricture on percutaneous or endoscopic cholangiography, and at least one of the following: Polysomy on fluorescent in situ hybridization-16; CA19.9 level >100 U/ml; malignant cytology or histology on transluminal brushings or biopsy; and identification of a hilar mass on cross-sectional imaging at the site of the malignant-appearing stricture (29). Based on this procedure, it is often difficult to distinguish malignant carcinoma from benign lesions, particularly in the setting of PSC. Nearly half of patients that underwent the Mayo
Protocol did not have pathological confirmation of carcinoma prior to neoadjuvant therapy and explanted liver pathological analyses revealed no tumor residue in a number of patients (29), thus raising the question as to whether the excellent result achieved in the Mayo Clinic protocol was attributed to the inclusion of patients with hilar benign lesions in treatment protocols. The authors explained that brachytherapy was responsible for dissolving the tumor, however, this is extremely unlikely as a complete pathological response with contemporary chemotherapy and radiation has rarely been reported (30). This phenomenon was validated in the current study; focal necrosis was observed, but the tumor did not disappear. An accurate pathological confirmation of diagnosis pretreatment may therefore be necessary to improve long-term survival rates of patients. 
Table I. Course of serum TB, DB and ALT post-OLT.

\begin{tabular}{lcccrrr}
\hline $\begin{array}{l}\text { Biological } \\
\text { features }\end{array}$ & $\begin{array}{c}\text { Normal } \\
\text { range }\end{array}$ & $\begin{array}{c}\text { Prior to } \\
\text { OLT }\end{array}$ & $\begin{array}{c}\text { Day } 3 \\
\text { post-OLT }\end{array}$ & $\begin{array}{c}\text { Day } 9 \\
\text { post-OLT }\end{array}$ & $\begin{array}{c}\text { Day 14 } \\
\text { post-OLT }\end{array}$ & $\begin{array}{c}\text { Day 25 } \\
\text { post-OLT }\end{array}$ \\
\hline ALT, U/1 & $0-40$ & 95.8 & 575.9 & 64.4 & 42.3 & 50.1 \\
post-OLT
\end{tabular}

TB, total bilirubin; DB, direct bilirubin; OLT, orthotopic liver transplantation; ALT, alanine aminotransferase; AST, aspartate aminotransferase; ALP, alkaline phosphatase; GGT, $\gamma$-glutamyl transferase; Alb, albumin.

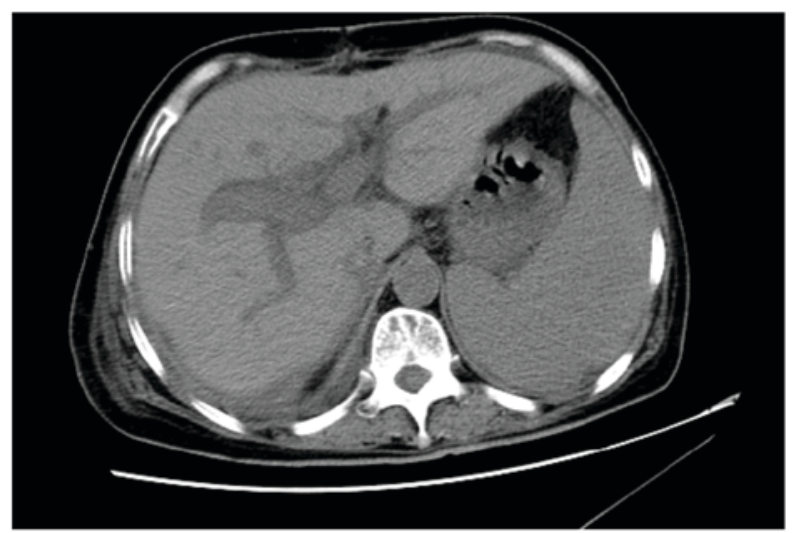

Figure 5. Computed topography image demonstrating no signs of tumor recurrence 2 months post-orthotopic liver transplantation.

Conventional percutaneous or endoscopic fine aspiration leads to tumor seeding and therefore cannot be employed to diagnose hilar cholangiocarcinoma. Thus, in the current study, ERCP was performed to obtain brush cytology, which was examined by a pathologist at the same time. Repeated brush was performed until the pathological diagnosis was confirmed. This approach improved the sensitivity and specificity of diagnosis.

Brachytherapy has been included in the majority of previous neoadjuvant protocols. Nebraska University employed Ir-192 wires for tumor irradiation through percutaneous biliary catheters, whereas the Mayo Clinic harnessed Ir-192 seeds loaded in the stent by $\operatorname{ERCP}(23,29)$. Brachytherapy was administered as an intensified radiation method in each strategy, which required withdrawal after $24 \mathrm{~h}$. The current study used I-125 seeds as a radiation source, as it was considered that continued and effective brachytherapy would minimize tumor progression prior to transplantation. This hypothesis was based on the findings of previous studies, which demonstrated that treating advanced tumors around the pancreatic head region using I-125 radioactive stents resulted in $72.7 \%$ of patients having 'stable disease' 2 months after treatment, which is remarkable regarding the high malignancy and advanced stage of the tumors $(31,32)$. Consistent with these results, in the current study, the image monitor and tumor marker profile exhibited no signs of recurrence in the patient.
Identifying ways to avoid tumor recurrence following transplantation is an important clinical goal. The majority of liver transplantation centers include corticosteroid treatment in their anti-rejection regimen, which is associated with tumor recurrence and major adverse events. However, a recent meta-analysis demonstrated that steroid-free tacrolimus-based immunosuppression was safe and that certain adverse events, including diabetes and hepatitis $\mathrm{C}$ virus recurrence, occur less frequently in the patients that received corticosteroids following liver transplantation (33). Therefore, early withdrawal of steroids may minimize the chance of recurrence. Rapamycin, a novel immunosuppressive agent, has been reported to inhibit metastatic tumor growth by anti-angiogenesis (34). The antitumor properties have been validated by its effectiveness in treating patients with hepatic cell carcinoma (HCC) (35). Compared with conventional tacrolimus, rapamycin significantly reduces the probability of tumor recurrence (36). Further studies are required to investigate the effectiveness of rapamycin treatment in hilar cholangiocarcinoma following liver transplantation.

The experience of the present study highlights a number of issues to be considered prior to the initiation of neoadjuvant therapy. Firstly, pathological confirmation of hilar cholangiocarcinoma must be obtained, as these patients may benefit from the neoadjuvant protocol. The most effective way of achieving this is by cytological brush through ERCP. Secondly, brachytherapy serves a pivotal role in the neoadjuvant protocol. Among the various methods of brachytherapy, the use of an I-125 radioactive stent may be the most effective method. Thirdly, in order to reduce tumor recurrence following liver transplantation, early steroid withdrawal or steroid-free liver transplantation should be considered in combination with rapamycin usage in the post-operative stage.

The present case demonstrates that neoadjuvant plus OLT may be an effective method of treating hilar cholangiocarcinoma, particularly in the presence of unresectable tumors. Following this treatment, the current patient was alive and disease-free for 8 months. In conclusion, the following issues should be considered before initiating neoadjuvant therapy. In light of the complexity and expense, it is necessary to obtain pathological confirmation of hilar cholangiocarcinoma. Patients diagnosed with cholangiocarcinoma can benefit from the neoadjuvant protocol discussed in the present study. Brush cytology using 
ERCP is an effective and rapid way of diagnosing cholangiocarcinoma. Brachytherapy serves a pivotal role in the neoadjuvant protocol. Of the various methods of brachytherapy, I-125 radioactive stent may present the best solution. To reduce recurrence following liver transplantation as much as possible, early steroid withdrawal from the immunosuppression protocols could be considered in combination with administration of rapamycin.

\section{Competing interests}

The authors declare that they have no competing interests.

\section{References}

1. Khan SA, Toledano MB and Taylor-Robinson SD: Epidemiology, risk factors, and pathogenesis of cholangiocarcinoma. HPB (Oxford) 10: 77-82, 2008.

2. Skipworth JR, Keane MG and Pereira SP: Update on the management of cholangiocarcinoma. Dig Dis 32: 570-578, 2014

3. Hemming AW, Reed AI, Fujita S, Foley DP and Howard RJ: Surgical management of hilar cholangiocarcinoma. Ann Sur 241: 693-702, 2005.

4. Guthrie CM, Banting SW, Garden OJ and Carter DC: Segment III cholangiojejunostomy for palliation of malignant hilar obstruction. Br J Surg 81: 1639-1641, 1994.

5. Hassoun Z, Gores GJ and Rosen CB: Preliminary experience with liver transplantation in selected patients with unresectable hilar cholangiocarcinoma. Surg Oncol Clin N Am 11: 909-921, 2002.

6. Petrowsky $\mathrm{H}$ and Hong JC: Current surgical management of hilar and intrahepatic cholangiocarcinoma: The role of resection and orthotopic liver transplantation. Transplant Proc 41: 4023-4035, 2009.

7. Ghali P, Marotta PJ, Yoshida EM, Bain VG, Marleau D, Peltekian K, Metrakos P and Deschênes M: Liver transplantation for incidental cholangiocarcinoma: Analysis of the Canadian experience. Liver Transpl 11: 1412-1416, 2005.

8. Robles R, Figueras J, Turrion VS, Margarit C, Moya A, Varo E, Calleja J, Valdivieso A, Valdecasas JC, López P, et al: Spanish experience in liver transplantation for hilar and peripheral cholangiocarcinoma. Ann Surg 239: 265-271, 2004.

9. Meyer CG, Penn I and James L: Liver transplantation for cholangiocarcinoma: Results in 207 patients. Transplantation 69: $1633-1637,2000$.

10. Rea DJ, Heimbach JK, Rosen CB, Haddock MG, Alberts SR, Kremers WK, Gores GJ and Nagorney DM: Liver transplantation with neoadjuvant chemoradiation is more effective than resection for hilar cholangiocarcinoma. Ann Surg 242: 451-461, 2005.

11. Gores GJ, Gish RG, Sudan D and Rosen CB MELD Exception Study Group: Model for end-stage liver disease (MELD) exception for cholangiocarcinoma or biliary dysplasia. Liver Transpl (12 Suppl 3): S95-S97, 2006.

12. Darwish Murad S, Kim WR, Harnois DM, Douglas DD, Burton J, Kulik LM, Botha JF, Mezrich JD, Chapman WC, Schwartz JJ, et al: Efficacy of neoadjuvant chemoradiation, followed by liver transplantation, for perihilar cholangiocarcinoma at 12 US centers. Gastroenterology 143: 88-98. e3; quiz e14, 2012.

13. Paul A, Kaiser GM, Molmenti EP, Schroeder T, Vernadakis S, Oezcelik A, Baba HA, Cicinnati VR and Sotiropoulos GC: Klatskin tumors and the accuracy of the Bismuth-Corlette classification. Am Surg 77: 1695-1699, 2011.

14. Razumilava N and Gores GJ: Classification, diagnosis, and management of cholangiocarcinoma. Clin Gastroenterol Hepatol 11: 13-21.e1; quiz e3-e4, 2013.

15. Todoroki T, Kawamoto T, Koike N, Takahashi H, Yoshida S, Kashiwagi H, Takada Y, Otsuka M and Fukao K: Radical resection of hilar bile duct carcinoma and predictors of survival. Br J Surg. 87: 306-313, 2000.

16. Klempnauer J, Ridder GJ, von Wasielewski R, Werner M, Weimann A and Pichlmayr R: Resectional surgery of hilar cholangiocarcinoma: A multivariate analysis of prognostic factors. J Clin Oncol 15: 947-954, 1997.

17. Nakeeb A, Pitt HA, Sohn TA, Coleman J, Abrams RA, Piantadosi S, Hruban RH, Lillemoe KD, Yeo CJ and Cameron JL: Cholangiocarcinoma. A spectrum of intrahepatic, perihilar, and distal tumors. Ann Surg 224: 463-465, 1996.
18. Wu Y, Johlin FC, Rayhill SC, Jensen CS, Xie J, Cohen MB and Mitros FA: Long-term, tumor-free survival after radiotherapy combining hepatectomy-Whipple en bloc and orthotopic liver transplantation for early-stage hilar cholangiocarcinoma. Liver Transpl 14: 279-286, 2008

19. Neuhaus P, Jonas S, Bechstein WO, Lohmann R, Radke C, Kling N, Wex C, Lobeck H and Hintze R: Extended resections for hilar cholangiocarcinoma. Ann Surg 230: 808-819, 1999.

20. Alessiani M, Tzakis A, Todo S, Demetris AJ, Fung JJ and Starzl TE: Assessment of five-year experience with abdominal organ cluster transplantation. J Am Coll Surg 180: 1-9, 1995.

21. Zardavas D and Piccart M: Neoadjuvant therapy for breast cancer. Ann Rev Med 66: 31-48, 2015.

22. Kelsen D: Neoadjuvant therapy for gastrointestinal cancers. Oncol (Williston Park) 7: 25-32-41, 1993.

23. Sudan D, DeRoover A, Chinnakotla S, Fox I, Shaw B Jr, McCashland T, Sorrell M, Tempero M and Langnas A: Radiochemotherapy and transplantation allow long-term survival for nonresectable hilar cholangiocarcinoma. Am J Transplant 2: 774-779, 2002.

24. Darwish Murad S, Kim WR, Therneau T, Gores GJ, Rosen CB, Martenson JA, Alberts SR and Heimbach JK: Predictors of pretransplant dropout and posttransplant recurrence in patients with perihilar cholangiocarcinoma. Hepatology 56: 972-981, 2012.

25. Hong JC, Jones CM, Duffy JP, Petrowsky H, Farmer DG, French S, Finn R, Durazo FA, Saab S, Tong MJ, et al: Comparative analysis of resection and liver transplantation for intrahepatic and hilar cholangiocarcinoma: A 24-year experience in a single center. Arch Surg 146: 683-689, 2011

26. Gringeri E, Bassi D, D'Amico FE, Boetto R, Polacco M, Lodo E, D'Amico F, Vitale A, Boccagni P, Zanus G and Cillo U: Neoadjuvant therapy protocol and liver transplantation in combination with pancreatoduodenectomy for the treatment of hilar cholangiocarcinoma occurring in a case of primary sclerosing cholangitis: Case report with a more than 8-year disease-free survival. Transplant Proc 43: 1187-1189, 2011.

27. Schule S, Altendorf-Hofmann A, Utess F, Uteß F, Rauchfuß F, Freesmeyer M, Knösel T, Dittmar Y and Settmacher U: Liver transplantation for hilar cholangiocarcinoma--a single-centre experience. Langenbecks Arch Surg 398: 71-77, 2013.

28. Gu J, Bai J, Shi X, Zhou J, Qiu Y, Wu Y, Jiang C, Sun X, Xu F, Zhang Y and Ding Y: Efficacy and safety of liver transplantation in patients with cholangiocarcinoma: A systematic review and meta-analysis. Int J Cancer 130: 2155-2163, 2012.

29. Heimbach JK, Gores GJ, Haddock MG, Alberts SR, Pedersen R, Kremers W, Nyberg SL, Ishitani MB and Rosen CB: Predictors of disease recurrence following neoadjuvant chemoradiotherapy and liver transplantation for unresectable perihilar cholangiocarcinoma. Transplantation 82: 1703-1707, 2006.

30. Kelley RK, Hirose R and Venook AP: Can we cure cholangiocarcinoma with neoadjuvant chemoradiation and liver transplantation? Time for a multicenter trial. Liver Transpl 18: 509-513, 2012.

31. Liu Y, Liu JL, Cai ZZ, Lu Z, Gong YF, Wu HY, Man XH, Jin ZD and Li ZS: A novel approach for treatment of unresectable extrahepatic bile duct carcinoma: design of radioactive stents and an experimental trial in healthy pigs. Gastrointest Endosc 69: 517-524, 2009.

32. Liu Y, Lu Z, Zou DW, Jin ZD, Liu F, Li SD, Zhan XB, Zhang WJ, Wu RP, Yao YZ, et al: Intraluminal implantation of radioactive stents for treatment of primary carcinomas of the peripancreatic-head region: a pilot study. Gastrointest Endosc 69: 1067-1073, 2009.

33. Sgourakis G, Radtke A, Fouzas I, Mylona S, Goumas K, Gockel I, Lang $\mathrm{H}$ and Karaliotas C: Corticosteroid-free immunosuppression in liver transplantation: a meta-analysis and meta-regression of outcomes. Transpl Int 22: 892-905, 2009.

34. Guba M, von Breitenbuch P, Steinbauer M, Koehl G, Flegel S, Hornung M, Bruns CJ, Zuelke C, Farkas S, Anthuber M, et al: Rapamycin inhibits primary and metastatic tumor growth by antiangiogenesis: involvement of vascular endothelial growth factor. Nat Med 8: 128-135, 2002.

35. Bruix J, Sherman M; Practice Guidelines Committee, American Association for the Study of Liver Diseases: Management of hepatocellular carcinoma. Hepatology 42: 1208-1236, 2005.

36. Klintmalm G and O'Farrelly C: Taking the rap: Multiple effects of blocking mammalian target of rapamycin. Hepatology 57: 1-3, 2013.

This work is licensed under a Creative Commons Attribution-NonCommercial-NoDerivatives 4.0 International (CC BY-NC-ND 4.0) License. 